\title{
The transverse velocity and excitation structure of the HH 110 jet $^{\star}$
}

\author{
A. Riera ${ }^{1,2, \star \star}$, R. López ${ }^{2}$, A. C. Raga ${ }^{3}$, R. Estalella ${ }^{2}$, and G. Anglada ${ }^{4}$ \\ 1 Departament de Física i Enginyeria Nuclear, Universitat Politècnica de Catalunya, Av. Víctor Balaguer s/n, \\ 08800 Vilanova i la Geltrú, Spain \\ 2 Departament d'Astronomia i Meteorologia, Universitat de Barcelona, Av. Diagonal 647, 08028 Barcelona, Spain \\ e-mail: robert, rosario@am.ub.es \\ 3 Instituto de Ciencias Nucleares, Universidad Nacional Autónoma de México, Apartado Postal 70-543, \\ 04510 México D.F., México \\ e-mail: raga@astroscu.unam.mx \\ 4 Instituto de Astrofísica de Andalucía, CSIC, Camino Bajo de Huétor 24, 18008 Granada, Spain \\ e-mail: guillem@iaa.es
}

Received 23 October 2002 / Accepted 16 December 2002

\begin{abstract}
We present long-slit spectroscopic observations of the HH 110 jet obtained with the $4.2 \mathrm{~m}$ William Herschel Telescope. We have obtained for the first time, spectra for slit positions along and across the jet axis (at the position of knots B, $\mathrm{C}, \mathrm{I}, \mathrm{J}$ and $\mathrm{P}$ ) to search for the observational signatures of entrainment and turbulence by studying the kinematics and the excitation structure. We find that the HH 110 flow accelerates from a velocity of $35 \mathrm{~km} \mathrm{~s}^{-1}$ in knot A up to $110 \mathrm{~km} \mathrm{~s}^{-1}$ in knot $\mathrm{P}$. We find some systematic trends for the variation of the emission line ratios along the jet. No clear trends for the variation of the radial velocity are seen across the width of the jet beam. The cross sections of the jet show complex radial velocity and line emission structures which differ quite strongly from each other.
\end{abstract}

Key words. ISM: individual: HH 110 - ISM: jets and outflows - stars: pre-main sequence

\section{Introduction}

The HH 110 jet is found in L1647, in the Orion B cloud complex. There is some evidence that this object corresponds to the early stages of a jet-cloud collision. The jet presents a rather complex morphology in $\mathrm{H} \alpha$ and [S II] images (Reipurth \& Olberg 1991), with noticeable wiggles along its length, being suggestive of a turbulent flow. This object shows a very different morphology in near-infrared images (Davis et al. 1994; Noriega-Crespo et al. 1996). In the molecular hydrogen lines, the jet is nearly straight and the molecular emission appears shifted westward relative to the optical emission. The morphological differences and the displacement between the optical and near infrared emissions have been interpreted as the result of a grazing collision between the jet and a dense molecular

Send offprint requests to: A. Riera,

e-mail: angels.riera@upc.es

* Based on observations made with the $4.2 \mathrm{~m}$ William Herschel Telescope operated on La Palma by the Issac Newton Group of Telescopes at the Observatorio del Roque de los Muchachos of the Instituto de Astrofísica de Canarias.

$\star \star$ On sabbatical leave at the Instituto de Ciencias Nucleares, Universidad Nacional Autónoma de México, Apartado Postal 70-543, 04510 México D.F., México. cloud core. In this scenario, the jet strikes the molecular core and is deflected. The $\mathrm{H}_{2}$ emission is tracing the region where the atomic and molecular gas interact (Raga \& Cantó 1995). This proposed scenario is actually reinforced by observational evidence, and has recently been modeled in some detail (Raga et al. 2002).

The morphology of HH 110 first suggested that the driving jet source would be embedded in the dark lane, north of the apex of the flow. However, searches at optical, near infrared and radio continuum wavelengths have failed to detect a driving source along the HH 110 flow axis. Later on, Reipurth et al. (1996) reported the discovery of another jet, HH 270, located $3^{\prime}$ northeast of $\mathrm{HH} 110$. An embedded near infrared source, very close to IRAS $05489+0256$, is found along the HH 270 flow axis. Based on the morphology and on the direction of the proper motions of $\mathrm{HH} 270$ (which point toward the apex of HH 110), Reipurth et al. (1996) proposed that HH 270 and HH 110 are associated. The HH 270 flow, driven by the embedded source, could be having a grazing collision with a dense molecular clump, through which the jet is deflected into the HH 110 flow (Raga et al. 2002).

Further observational results also are in agreement with this scenario. Rodríguez et al. (1998) detected centimeter radio 
continuum emission at the position of the embedded near infrared source. This radio continuum source (VLA 1) appears sligthly elongated in the NE-SW direction, with the major axis aligned with the $\mathrm{HH} 270$ flow axis. This suggests that the VLA 1 source traces the base of the flow giving rise to the $\mathrm{HH}$ 270/HH 110 system. On the other hand, Sepúlveda (2001) detected, through ammonia mapping, a high density clump of molecular gas which is spatially associated with the $\mathrm{HH} 110$ flow, being located in the region where $\mathrm{HH} 270$ abruptly changes its direction to emerge as $\mathrm{HH} 110$. This high density gas, traced by the ammonia emission, could be responsible for the deflection of the HH 270 flow.

Choi (2001) has obtained $\mathrm{HCO}^{+}$interferometric maps, and does not detect emission in the crossing point of the $\mathrm{HH} 270$ and $\mathrm{HH} 110$ flow axes. Choi points out that this result is in apparent contradiction with the "deflected jet" interpretation of these flows. However, Raga et al. (2002) suggest that this result is not necessarily in contradiction with the deflected jet model, since the impact point might have moved into the dense cloud, so that it would now lie to the West of the HH 110 axis (where Choi 2001 does detect extended $\mathrm{HCO}^{+}$emission).

The HH 110 jet seems to be suitable for searching for the observational signatures of entrainment and turbulence (as predicted by theoretical models) by studying the kinematics and the excitation structure along and across the jet axis. In this paper, we present the results obtained from long-slit, high-resolution spectroscopy of the HH 110 jet. We have obtained spectra for slit positions both along and across the jet beam, allowing us to measure the spatially resolved radial velocities and emission line ratios along and across the jet. The observations are described in Sect. 2, and the results are presented in Sect. 3. Finally, the results are discussed in Sect. 4.

The data discussed in this paper represent a significant step forward with respect to previous spectroscopic observations of $\mathrm{HH}$ 110. As far as we are aware, the only published spectra of this object are the low resolution spectrum of Reipurth \& Olberg (1991) and the high-resolution spectrum of Reipurth et al. (1996), both corresponding to a single slit position along the outflow axis. We are now presenting high-resolution spectroscopy with substantially more extensive spatial coverage, consisting of several slit positions both along and across the outflow axis. Throughout the paper, we attempt to compare our results (at the appropriate spatial positions) with the ones obtained from the spectrum of Reipurth et al. (1996), and the line intensities obtained from our spectra with the narrow band images of Reipurth et al. (1996) and Noriega-Crespo et al. (1996).

\section{Observations}

Long-slit, high-resolution spectra of the HH 110 jet were obtained during the nights of December 12 and 13, 1998. The red arm of the double-armed spectrograph ISIS (Carter et al. 1994) and a Tektronics CCD detector of $1024 \times 1024$ pixels (pixel size of $24 \mu \mathrm{m}$ ) were used at the Cassegrain focus of the $4.2 \mathrm{~m}$ William Herschel Telescope (WHT) at the Observatorio del Roque de los Muchachos (La Palma, Spain). The highresolution grating R1200R (dispersion $17 \AA \mathrm{mm}^{-1}$ ) centered at $6600 \AA$ was employed, covering the $\mathrm{H} \alpha$, [N II] 6548 ,
Table 1. Journal of observations.

\begin{tabular}{lcccc}
\hline \hline Date & Knot & $\begin{array}{c}\text { P.A. } \\
\text { (degrees) }\end{array}$ & Slit Position $^{1}$ & $\begin{array}{c}\text { Exp. Time } \\
\text { (s) }\end{array}$ \\
\hline Dec. 12 & A-Q & 13.6 & sa & 1800 \\
& B & 103.6 & sb & 3600 \\
& C & 103.6 & sc & 3600 \\
& I-J & 103.6 & sd & 3600 \\
Dec. 13 & A-Q & 13.6 & sa & 1800 \\
& I-J & 103.6 & sd & 1800 \\
& P-Q & 103.6 & se & 5400 \\
\hline
\end{tabular}

${ }^{1}$ See Fig. 1.

$6583 \AA$ and [S II] 6717, $6731 \AA$ A lines and providing a spectral sampling of $0.41 \AA \operatorname{pixel}^{-1}$ (equivalent to $20 \mathrm{~km} \mathrm{~s}^{-1}$ at $\mathrm{H} \alpha$ ). The spatial sampling was $0.36^{\prime \prime}$ pixel $^{-1}$ and the seeing had a $F W H M$ of $2^{\prime \prime}$ to $3^{\prime \prime}$. The spectrograph slit had a projected width of $1^{\prime \prime} .5$.

Spectra of the HH 110 jet were obtained at different positions: in one of these positions, the slit was placed along the central axis of the jet. For the rest of the spectra, the slit was positioned perpendicular to the jet axis and each of these spectra was obtained cutting the jet beam across a bright knot (see Table 1 and Fig. 1). This means that, for the first time, we were able to study the kinematic and excitation structure across the flow.

The spectra were reduced using the standard tasks for long-slit spectroscopy within the IRAF package ${ }^{1}$, that includes bias substraction, flat-fielding correction, wavelength calibration and sky subtraction. The spectra were corrected for cosmic ray events by median filtering several exposures obtained at the same slit position. The spectra were not flux calibrated.

The results obtained from these observations are described in the following section.

\section{Results}

\subsection{Structure along the $\mathrm{HH} 110$ jet}

In Fig. 2, we present the H $\alpha$ and [S II] $6717 \AA$ PV (positionvelocity) diagrams along the jet axis. We have used the nomenclature of Reipurth \& Olberg (1991) for the knots, the most prominent of which are identified in Fig. 2. The radial velocities are relative to the ambient molecular cloud which has a heliocentric radial velocity of $23 \mathrm{~km} \mathrm{~s}^{-1}$ (Reipurth \& Olberg 1991). Our PV diagrams are consistent with the [S II] PV diagram of Reipurth et al. (1996).

Figure 2 shows a change in radial velocity along knot A, with a decrease of $25 \mathrm{~km} \mathrm{~s}^{-1}$ (from -48 to $-23 \mathrm{~km} \mathrm{~s}^{-1}$ ) in $1^{\prime \prime}$.7. Such an effect was already noted by Reipurth et al. (1996). From Fig. 2, we also see that the FWHM of the [S II] $6717 \AA$ emission line has the highest values at knots B and $\mathrm{C}$, with a value of $\sim 90 \mathrm{~km} \mathrm{~s}^{-1}$, and decreases to a value of $70 \mathrm{~km} \mathrm{~s}^{-1}$ at knot $\mathrm{H}$, in clear agreement with the results of

\footnotetext{
${ }^{1}$ IRAF is distributed by the National Optical Astronomy Observatories, which is operated by the Association of Universities for Research in Astronomy, Inc., under a contract agreement with the National Science Foundation.
} 


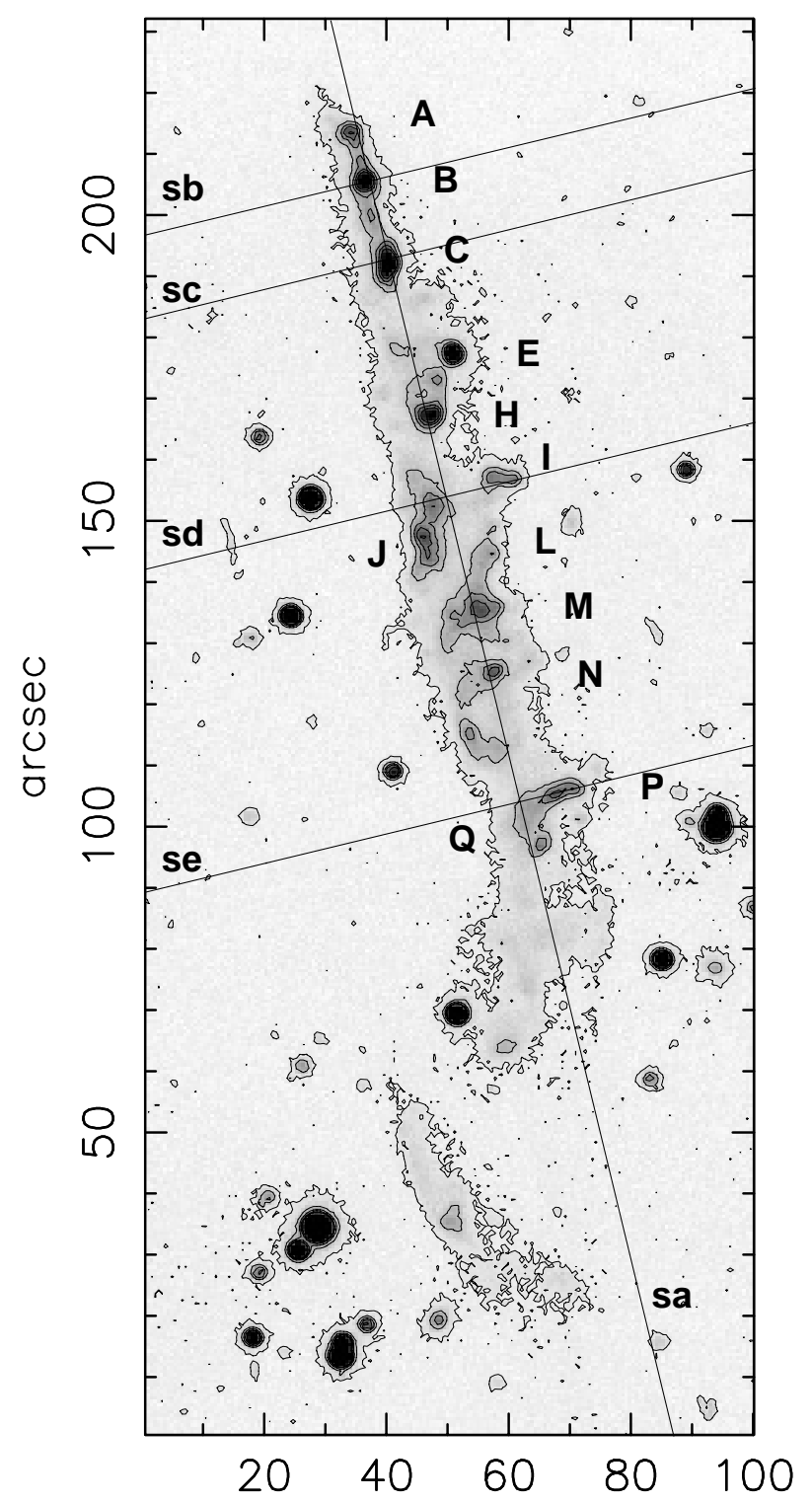

$\operatorname{arcsec}$

Fig. 1. [S II] $6717+6731$ image of HH 110 obtained by the authors on Dec. 1993 at the $2.5 \mathrm{~m}$ Isaac Newton Telescope (INT) at the Observatorio del Roque de los Muchachos. On this image, the slit positions used for the spectroscopy described in the paper have been indicated. North is up and East is to the left.

Reipurth et al. (1996). Beyond knot H, the line width remains approximately constant. We should note that these line widths are well resolved with our $30 \mathrm{~km} \mathrm{~s}^{-1}$ effective spectral resolution.

Figure 3 shows the radial velocities (with respect to the molecular cloud) corresponding to the peaks of the $\mathrm{H} \alpha$ and [S II] $6717 \AA$ line profiles along the HH 110 jet. All knots show blueshifted emission lines, with relatively small radial velocity variations along the axis of the jet.

The radial velocity of knot $\mathrm{B}$ is of $\sim-25 \mathrm{~km} \mathrm{~s}^{-1}$, and it increases (in modulus) at the position of knot $\mathrm{C}$ to $\sim-43 \mathrm{~km} \mathrm{~s}^{-1}$ in [S II] (see Fig. 3). Knot $\mathrm{H}$ has a radial velocity of $\sim-50 \mathrm{~km} \mathrm{~s}^{-1}$. At the location of knot $\mathrm{M}$, the [S II] and $\mathrm{H} \alpha$ lines show a range of radial velocities, between $\sim-50 \mathrm{~km} \mathrm{~s}^{-1}$ and $-60 \mathrm{~km} \mathrm{~s}^{-1}$. Knot $\mathrm{N}$ has a radial velocity of $\sim-55 \mathrm{~km} \mathrm{~s}^{-1}$. Knot $\mathrm{P}$ shows a range of radial velocities from -65 to $-55 \mathrm{~km} \mathrm{~s}^{-1}$.

Finally, knot Q has a radial velocity of $-50 \mathrm{~km} \mathrm{~s}^{-1}$ in $\mathrm{H} \alpha$ and $-10 \mathrm{~km} \mathrm{~s}^{-1}$ in [S II]. This difference appears to be a result of the fact that at the position of knot Q, two components with different radial velocities are seen in both the $\mathrm{H} \alpha$ and [S II] line profiles. While in $\mathrm{H} \alpha$ the high radial velocity component is dominant, in the [S II] lines the low velocity component is much brighter, resulting in the low radial velocity determined from the $[\mathrm{S} \mathrm{II}]$ lines.

Figure 3 reveals that the variation of radial velocities with distance along HH 110 is qualitatively similar (with the exception of knot Q) for the $\mathrm{H} \alpha$ and [S II] emission lines. In both cases, the jet accelerates with distance along the jet from -20 to $-65 \mathrm{~km} \mathrm{~s}^{-1}$ (with respect to the molecular cloud).

In Fig. 4, we present the velocity integrated line ratios for various knots, as derived from the long-slit spectra. The [N II] $6583 / \mathrm{H} \alpha$, the [S II] $(6717+6731) / \mathrm{H} \alpha$, and the [S II] 6717 / 6731 emission line ratios are plotted as a function of position along the slit. The emission line ratios obtained at December 12 and 13 are similar to each other (implying that the slit positions probably were almost coincident). In Figs. 4 and 5 we show the average of both spectra (with error estimates based on the statistical error as well as on the differences between the two observations), and the following discussion is also based on the average of both spectra. For comparison, we have also included the results from Reipurth et al. (1996).

The [N II] $6583 / \mathrm{H} \alpha$ emission line ratio has a value of $\sim 0.12$ in the bright knot $\mathrm{A}$. This ratio increases in knot $\mathrm{B}$, and decreases further out at the position of knot $\mathrm{C}$. This ratio is $\sim 0.20$ at the position of knot $\mathrm{H}$, and it increases to $\sim 0.40$ at knot M. At the position of knots $\mathrm{N}$ and $\mathrm{P}$ the $[\mathrm{N} \mathrm{II}] / \mathrm{H} \alpha$ ratio has an approximately constant value $(\sim 0.25)$ and decreases beyond knot $\mathrm{P}$.

The trend of variation of the [N II] 6583 / H $\alpha$ emission line ratio with angular distance is consistent with the results of Reipurth et al. (1996). However, the values reported by Reipurth et al. (1996) are significantly lower than our values.

The overall trend of variation of the $[\mathrm{S} \mathrm{II}] / \mathrm{H} \alpha$ emission along the length of the slit shows an almost constant value for the brightest knots (knots A, B, and C), where the ratio is $\sim 0.50$. The $[\mathrm{S} \mathrm{II}] / \mathrm{H} \alpha$ emission line ratio rapidly increases along knots $\mathrm{H}$ and $\mathrm{M}$, to values of $\sim 0.80$. The $[\mathrm{S} \mathrm{II]} \mathrm{/} \mathrm{H} \alpha$ ratio decreases at larger distances (at the position of knots $\mathrm{N}, \mathrm{P}$ and Q).

Figure 4 illustrates the differences between our results and the $[\mathrm{S} \mathrm{II}] / \mathrm{H} \alpha$ ratio trend along the $\mathrm{HH} 110$ jet derived by Reipurth et al. (1996). We observe a rapid increase of the [S II] / H $\alpha$ ratio from knot B to the location of knots H-M, and a linear decrease beyond knot M, while Reipurth et al. (1996) obtained a slow increase from knot $\mathrm{B}$ to knot $\mathrm{M}$ and a rapid decrease beyond knot M. Moreover, our [S II] / $\mathrm{H} \alpha$ ratios are significantly higher than the values reported by Reipurth et al. (1996).

Figure 4 also shows the [S II] 6717 / 6731 emission line ratios along the HH 110 jet. A first glance at the [S II] 6717 / 6731 plot shows a rapid increase of this ratio (and, consequently, a 


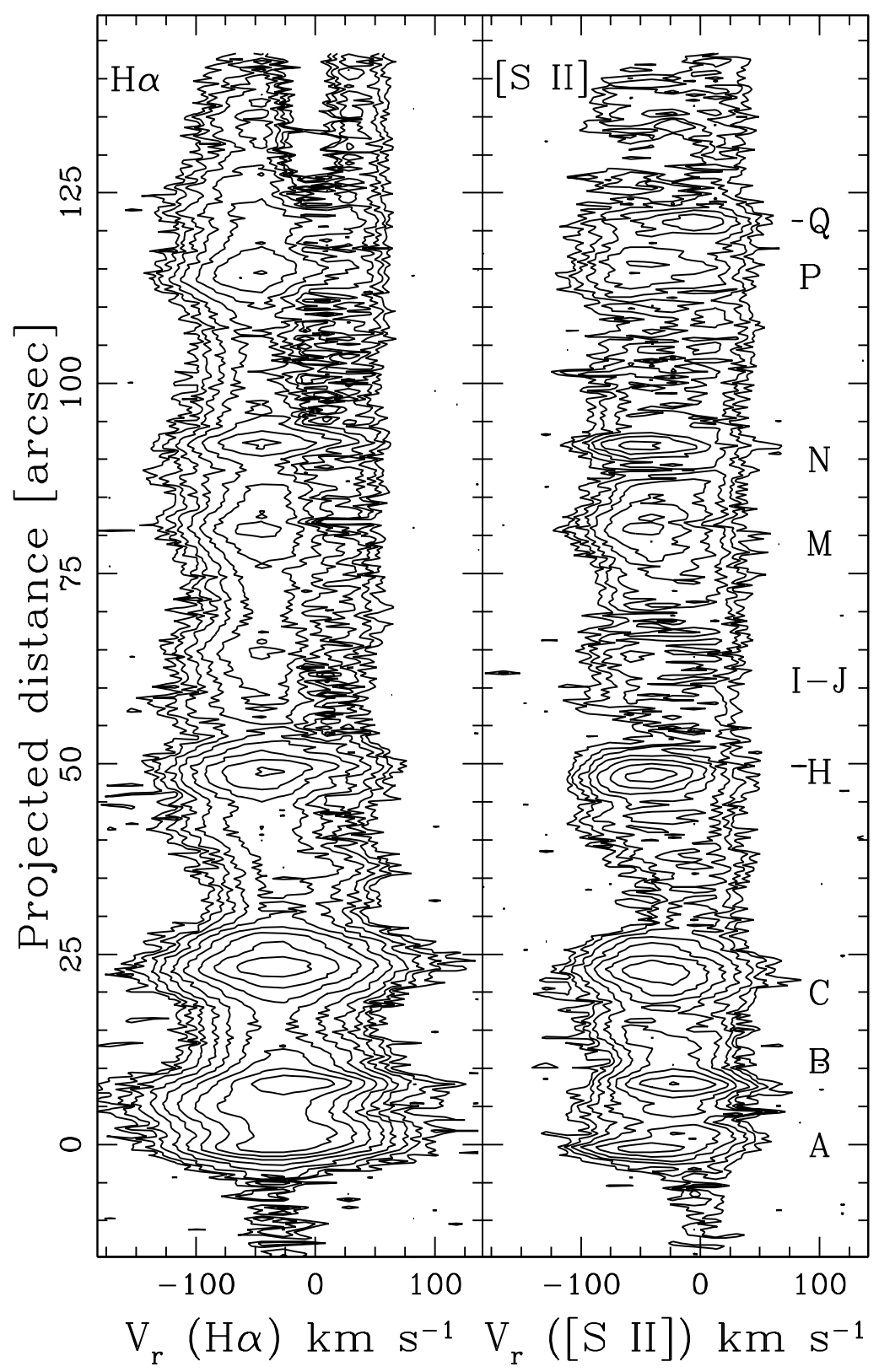

Fig. 2. H $\alpha$ and [S II] $6717 \AA$ A long slit spectra along the axis of the HH 110 jet (slit position "sa" in Fig. 1), depicted with factor of $2^{1 / 2}$, logarithmic contours. The radial velocities are relative to the ambient molecular cloud, which has a heliocentric radial velocity of $23 \mathrm{~km}^{-1}$ (see Reipurth \& Olberg 1991). The distances are measured from knot A.

rapid decrease of the electron density) with increasing distance along the jet. The electron density decreases from $\sim 865 \mathrm{~cm}^{-3}$ in knot A to $\sim 640 \mathrm{~cm}^{-3}$ in knot $\mathrm{B}$, with a lower value of $\sim 415 \mathrm{~cm}^{-3}$ in knot C. The [S II] $6717 / 6731$ ratio increases at knot $\mathrm{H}$, and consequently the electron density decreases to a value of $\sim 270 \mathrm{~cm}^{-3}$. The electron density increases at knots $\mathrm{M}$ and $\mathrm{N}$ (with values of $\sim 500 \mathrm{~cm}^{-3}$ ). The other knots show smaller electron densities $\left(\sim 150 \mathrm{~cm}^{-3}\right.$ in knot $\mathrm{P}$ and $\sim 50 \mathrm{~cm}^{-3}$ in knot Q).

The overall behaviour of the electron density along the jet axis is qualitatively similar to the measurements of Reipurth et al. (1996), who also found an almost continous decrease of the electron density along the axis of HH 110. However, our electron density values are somewhat lower than the values reported by Reipurth et al. (1996).
Finally, Fig. 5 illustrates the behaviour of the [N II] 6583 / [S II] $(6717+6731)$ (hereafter [N II] / [S II]) ratio (which is a measure of the excitation of the spectrum) along the axis of $\mathrm{HH} 110$. The [N II] / [S II] ratio shows a monotonic increase with distance from a value $\sim 0.20$ (at knot A) to $\sim 0.50$ (at knots $\mathrm{P}$ and $\mathrm{Q}$ ), with the exception of knot $\mathrm{B}$ which shows a $[\mathrm{N} \mathrm{II}] /[\mathrm{S} \mathrm{II}]$ line ratio of $\sim 0.45$, which lies well above the prevailing trend.

\subsection{Structure across the $H H 110$ jet: Knots $B$ and $C$}

First, we study the PV diagrams and the variation of the [N II] $6583 / \mathrm{H} \alpha$ and [S II] $(6717+6731) / \mathrm{H} \alpha$ emission line ratios and the electron density as a function of position across the jet at knots $\mathrm{B}$ and $\mathrm{C}$, which lie closer to the base of the 


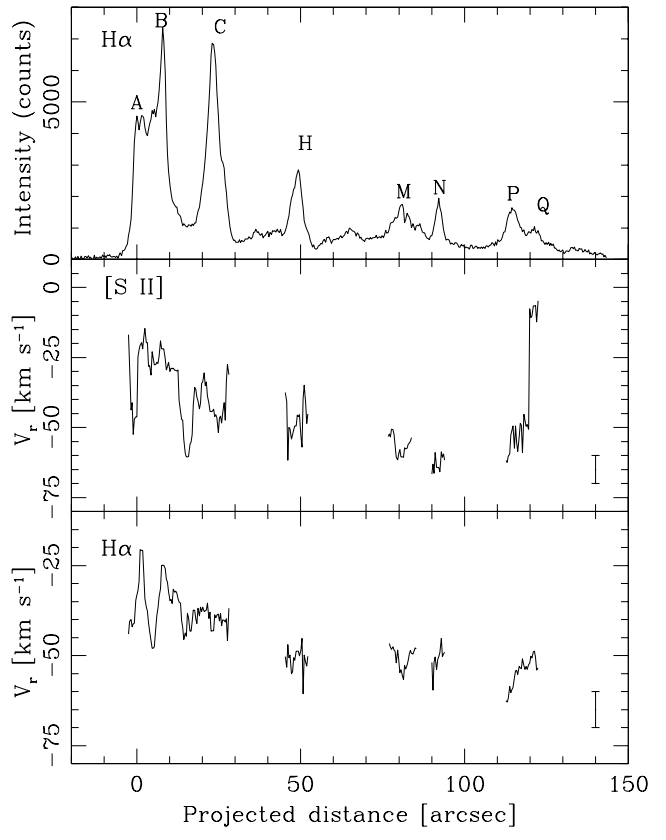

Fig. 3. Radial velocity corresponding to the peak of the $\mathrm{H} \alpha$ (bottom) and [S II] $6717 \AA$ line profiles (center) as a function of position along $\mathrm{HH}$ 110. Only the regions with a signal-to-noise ratio which is high enough to obtain a reliable determination of the radial velocities of the peak emission are shown. The radial velocities shown are relative to the ambient molecular cloud. The error bars in the central and bottom panels indicate typical errors in the radial velocities. The $\mathrm{H} \alpha$ spatial intensity distribution is also shown (top) in order to help with the identification of the knots. The distances are measured from knot A.

HH 110 jet than the other knots, and where the jet shows a well collimated morphology (see Fig. 1).

In Fig. 6 we present the PV diagrams across the beam of the jet. These measurements were obtained with spectrograph slit positions "sb" and "sc" (see Fig. 1 and Table 1), which intersect the jet at knots B and C, respectively. The distances were measured from the $\mathrm{H} \alpha$ intensity peak (located at 0.0 in the Fig. 6). We should remember that the molecular cloud is located towards the west (i.e., positive values of $y$ ).

The $\mathrm{H} \alpha$ and [S II] emission lines of knots B and $\mathrm{C}$ are characterised by the presence of a peak and a weak asymmetrical emission which extends eastwards. In the PV diagrams of knots B and C (see Fig. 6), we clearly see that the [S II] emission extends out to 6 " from the peak of the knot towards the west, and to $\sim 9^{\prime \prime}$ towards the east. We also see that the $\mathrm{H} \alpha$ emission extends $10^{\prime \prime}$ further out to the east. The secondary emission we have detected in $\mathrm{H} \alpha$ corresponds to the faint emission detected east of knots $\mathrm{B}$ and $\mathrm{C}$ in the $\mathrm{H} \alpha$ image obtained by Reipurth \& Olberg (1991, who labeled this region as X and Y).

The $\mathrm{H} \alpha$ and [S II] $6717 \AA$ A PV diagrams of knots B and C show an approximately constant radial velocity across the knots (see Fig. 6). The radial velocities (i.e. the mean values of the bright central regions of knots B and C) have values of $\sim-30 \mathrm{~km} \mathrm{~s}^{-1}$. These radial velocities are consistent within the errors with the velocity values derived for knots $B$ and $C$ from the spectra obtained along the axis of the jet (see Sect. 3.1). We see an increase of the radial velocity (in modulus) by $20 \mathrm{~km} \mathrm{~s}^{-1}$

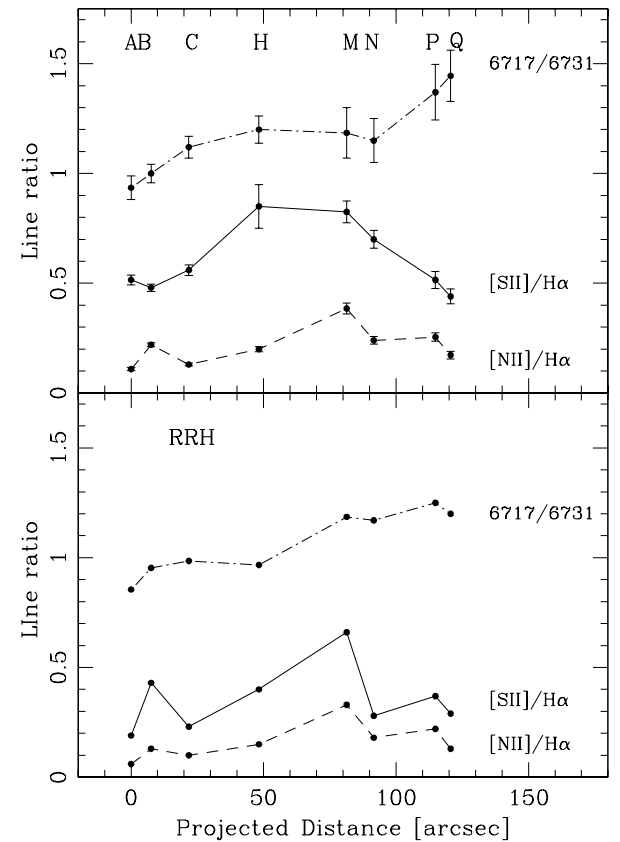

Fig. 4. The [N II] $6583 / \mathrm{H} \alpha$, the [S II] $(6717+6731) / \mathrm{H} \alpha$, and the [S II] 6717 / 6731 ratios computed from the velocity integrated line intensities are plotted for the knots intercepted by the slit position "sa". The top panel corresponds to the present data (where the two spectra obtained along the axis of the jet were averaged), and the bottom panel has been taken from Reipurth et al. (1996, labeled as RRH).

from the central part of knot B towards the edges (see Fig. 6), while no significant variation is observed across knot $C$.

Across knot B from west to east, the [N II] 6583 / $\mathrm{H} \alpha$ emission line ratio first increases from 0.20 to 0.30 in $\sim 3^{\prime \prime}$ and then rapidly decreases (see Fig. 7). The maximum of the [N II] / $\mathrm{H} \alpha$ ratio is displaced by $\sim 0$ ' 8 towards the west with respect the central peak of the $\mathrm{H} \alpha$ intensity.

The [S II] $(6717+6731) / \mathrm{H} \alpha$ ratio shows a similar, but less pronounced, variation across knot B (see Fig. 7). This ratio first increases from $\sim 0.40$ to 0.55 in $\sim 4^{\prime \prime}$, and then decreases eastwards to a value of $\sim 0.40$ at $-4^{\prime \prime}$ from the central position.

Knot B has an almost constant [S II] 6717 / 6731 line ratio of $\sim 0.95$ (see Fig. 7) across the central bright region, corresponding to a $n_{\mathrm{e}} \sim 700 \mathrm{~cm}^{-3}$. The density decreases at $y \sim \pm$ $2^{\prime \prime}$, where [S II] $6717 / 6731 \sim 1.17$ (e.g., $n_{\mathrm{e}} \sim 300 \mathrm{~cm}^{-3}$ ).

The [N II] / [S II] line ratio decreases across knot B from $\sim 0.60$ at $2^{\prime \prime}$ west of the central peak (i.e. $y=0$ ) to $\sim 0.25$ at $4^{\prime \prime}$ east of the central peak.

Across knot C, the [N II] 6583 / $\mathrm{H} \alpha$ emission line ratio is significantly lower than the corresponding values for knot $\mathrm{B}$, which is in agreement with the results obtained from the spectrum along the axis of the jet. The [N II] / $\mathrm{H} \alpha$ ratio slightly increases moving eastwards across the bright, central region (see Fig. 7), and then decreases further estwards. A larger variation across knot $\mathrm{C}$ was found for the [S II] $(6717+6731) / \mathrm{H} \alpha$ ratio, which increases across the central region (see Fig. 7) and then decreases further eastwards. The maximum of the [S II] / $\mathrm{H} \alpha$ distribution is located at $\sim 1$ ". 4 east of the $\mathrm{H} \alpha$ central peak.

At the central position of knot C, the [S II] 6717 / 6731 ratio is $\sim 1.08$ (see Fig. 7), and therefore the electron density has a 


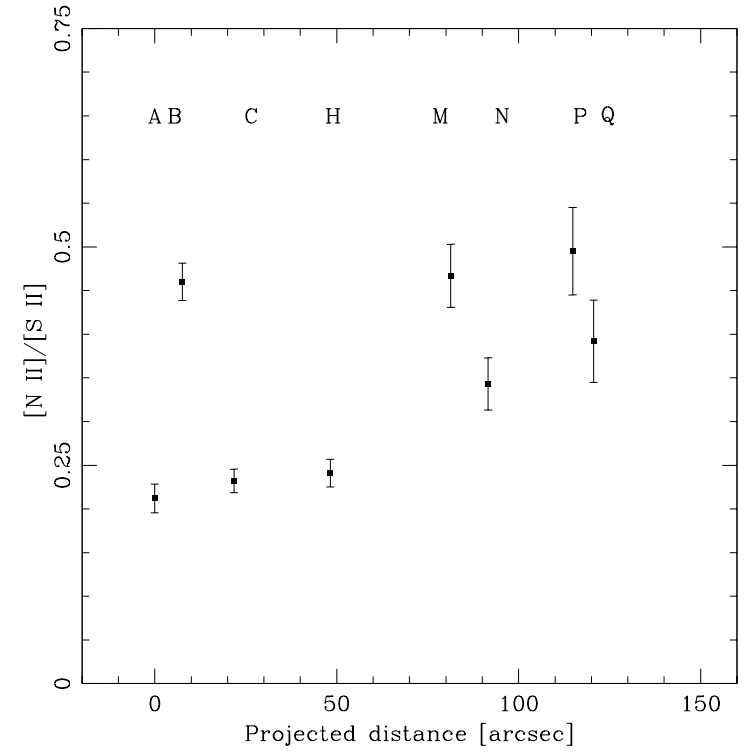

Fig. 5. The velocity integrated [N II] 6583 / [S II] (6717+6731) line ratio is plotted for the knots intercepted by the slit position "sa".

value of $\sim 450 \mathrm{~cm}^{-3}$. The [S II] $6717 / 6731$ ratio increases towards both sides, adopting a value $\sim 1.35$ at $\sim \pm 4$ " from the centre (i.e., $n_{\mathrm{e}} \sim 100 \mathrm{~cm}^{-3}$ ). The $[\mathrm{N} \mathrm{II}] /[\mathrm{S} \mathrm{II}]$ emission line ratio decreases across knot $\mathrm{C}$ from west to east, from $\sim 0.40$ to 0.20 across $5^{\prime \prime}$.

The results found in this section therefore are:

- the radial velocities of knots $\mathrm{B}$ and $\mathrm{C}$ are approximately constant across the width of the jet;

- the [N II] / H $\alpha$ and [S II] / $\mathrm{H} \alpha$ ratios show an increase across knots $\mathrm{B}$ and $\mathrm{C}$ moving from west to east (along $\sim 6^{\prime \prime}$ );

- the electron densities are constant in the brightest region of knots B and C, which are surrounded by lower density gas;

- the [N II] / [S II] emission line ratios across knots B and $\mathrm{C}$ show a similar trend, with a west to east decrease (i.e., a decrease of the excitation of the spectrum).

\subsection{Structure across $H H$ 110: Knots I, J, $P$ and $Q$}

At a position of $25^{\prime \prime}$ south of knot $\mathrm{A}$, the jet widens in a cone with a large opening angle. The slit positions "sd" and "se" (see Fig. 1 and Table 1) show emission extending $\sim 30$ " in [S II] cutting across knots I and J (slit position "sd"), and also across knot $\mathrm{P}$ (slit position "se"). The spectrum across knot $\mathrm{P}$ also shows emission arising from the northern edge of knot $\mathrm{Q}$ (see Fig. 1).

In Fig. 8 we present the PV diagrams across the beam of the jet at the position of knots I and J, and P and Q. Across knot J, we see a clear trend of decreasing radial velocities (in modulus) moving from west to east. In the western edge of knot $\mathbf{J}$ we measure a [S II] $6717 \AA$ radial velocity of $-55 \mathrm{~km} \mathrm{~s}^{-1}$, and a position $\sim 9^{\prime \prime}$ to the east this velocity has a value of $\sim-25 \mathrm{~km} \mathrm{~s}^{-1}$. In knot I, the peak of the [S II] $6717 \AA$ emission has a velocity of $\sim-70 \mathrm{~km} \mathrm{~s}^{-1}$, and the $\mathrm{H} \alpha$ emission has a radial velocity of $\sim-85 \mathrm{~km} \mathrm{~s}^{-1}$.
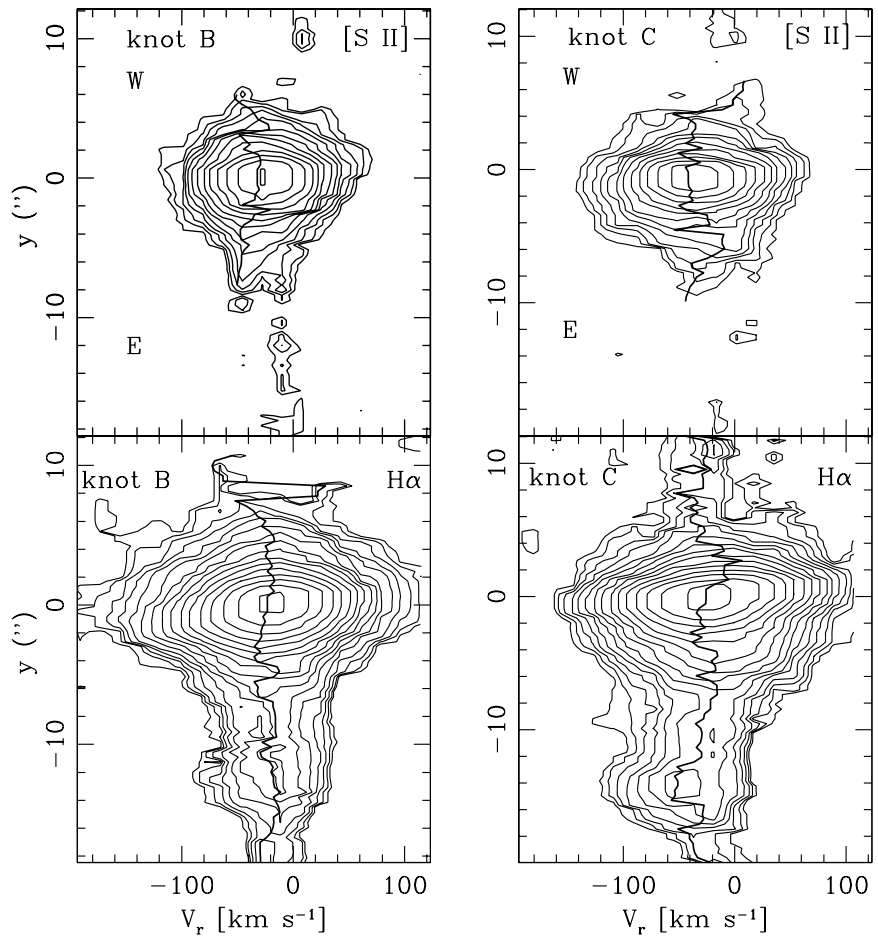

Fig. 6. $\mathrm{H} \alpha$ (bottom panels) and [S II] $6717 \AA$ (top panels) long slit spectrum across the jet at the positions of knots B (left panels) and C (right panels); depicted with $2^{1 / 2}$, logarithmic contours. The superimposed solid line is the radial velocity (with respect to the molecular cloud) corresponding to the peak of the line profile.

Figure 8 also shows the PV diagram across knots $\mathrm{P}$ and $\mathrm{Q}$. The $\mathrm{H} \alpha$ radial velocity as a function of position shows an S-shaped structure, with a peak value (in modulus) of $-67 \mathrm{~km} \mathrm{~s}^{-1}$ and a minimum value of $-40 \mathrm{~km} \mathrm{~s}^{-1}$. The peak of knot P (located at $y=0$ ) has a radial velocity of $-55 \mathrm{~km} \mathrm{~s}^{-1}$. The [S II] radial velocities have a similar dependence with position, but have values which are systematically less negative by $10 \mathrm{~km} \mathrm{~s}^{-1}$.

Figure 9 shows the $\left[\begin{array}{ll}\mathrm{N} & \mathrm{II}\end{array}\right] 6583 / \mathrm{H} \alpha$, [S II] $(6717+6731) / \mathrm{H} \alpha$, and [S II] 6717 / 6731 emission line ratios across knots $\mathrm{J}+\mathrm{I}$, and $\mathrm{P}+\mathrm{Q}$. The [N II] 6583 / H $\alpha$ ratio increases as a function of distance across knots I and $\mathrm{J}$ (from west to east). The [N II] $6583 / \mathrm{H} \alpha$ ratio increases across knot I (from west to east) up to a value of $\sim 0.30$. The [N II] $6583 / \mathrm{H} \alpha$ ratio rapidly decreases (from west to east) across knot J (see Fig. 9), decreasing from 0.30 to a value of 0.075 along 7 .' 0 .

The [S II] $(6717+6731) / \mathrm{H} \alpha$ line ratio also shows a linear increase across knot I and across the gas between the two knots (increasing from 0.30 to 0.65 over $12^{\prime \prime}$, see Fig. 9). From the western to the eastern edge of knot J, the [S II] / $\mathrm{H} \alpha$ ratio decreases from 0.6 to 0.2 .

The [N II] / [S II] emission line ratio changes abruptly at the position of the $\mathrm{H} \alpha$ peak of knot I, showing a value of $\sim 0.20$ to the west and $\sim 0.40$ to the east. At the position of knot $\mathrm{J}$, the [N II] / [S II] ratio has a constant value of $\sim 0.45$.

The [S II] 6717 / 6731 line ratio shows an overall increasing trend from west to east (see Fig. 9). The electron density 


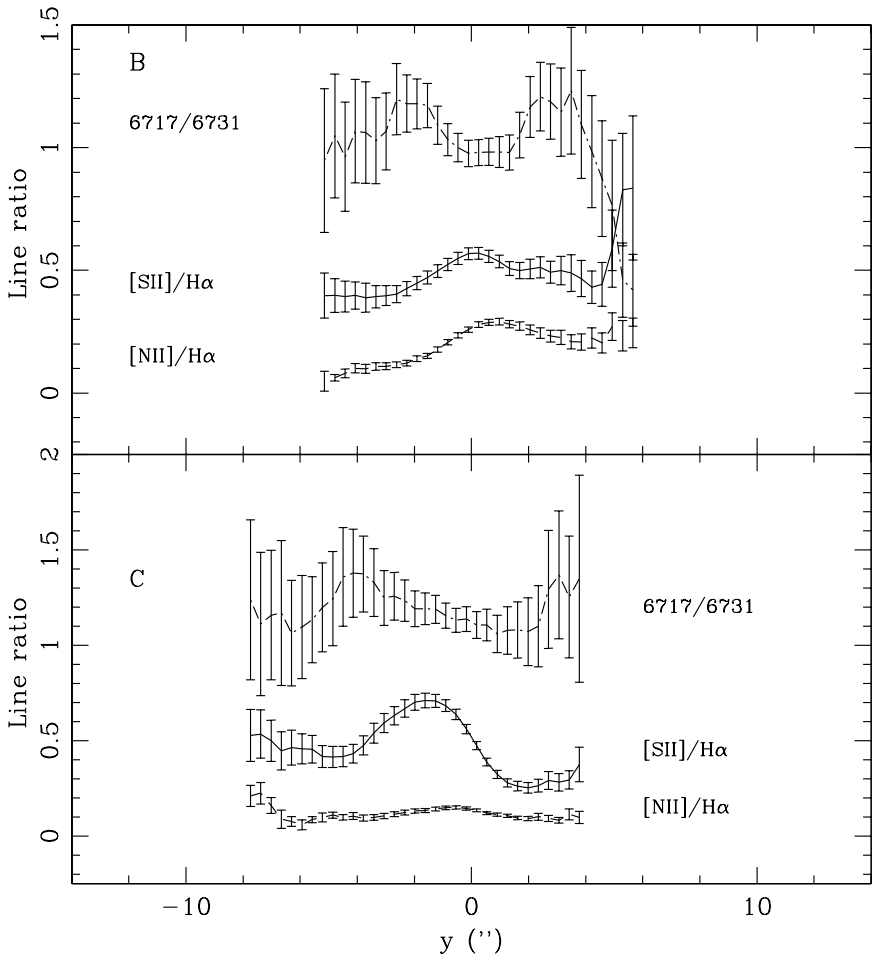

Fig. 7. [N II] $6583 / \mathrm{H} \alpha$, [S II] $(6717+6731) / \mathrm{H} \alpha$, and [S II] 6717 / 6731 line ratios through the cross section of the jet at the positions of knots B (top panel), and C (bottom panel), as a function of distance from the central peak. The intensities were integrated over all the radial velocity range in which line emission was detected.

has a mean value $\leq 100 \mathrm{~cm}^{-3}$ at the position of knot I. We see a W-E trend of increasing [S II] 6717/6731 line ratio across knot $\mathrm{J}$, with electron densities decreasing from $\sim 100 \mathrm{~cm}^{-3}$ to $<60 \mathrm{~cm}^{-3}$.

Figure 9 also illustrates the variation of the [N II] 6583 / H $\alpha$ ratio as a function of position across knot $\mathrm{P}$. Along the western edge of knot P, from west to east, the [N II] / H $\alpha$ ratio shows two peaks. The higher peak is displaced $\sim 3$ " to the west of the central part of knot $\mathrm{P}$. The lower peak approximately coincides with knot Q (even though this knot does not lie right on the position of our spectrograph slit, see Fig. 1).

The [S II] / H $\alpha$ ratio rapidly decreases from west to east across knot $\mathrm{P}$ from a value $>1.3$ (at the western edge) to 0.7 (at the location of the $\mathrm{H} \alpha$ maximum, see Fig. 9). Across knot Q, the [S II] / H $\alpha$ ratio is $\sim 0.9$, and smoothly decreases to the east of the knot.

The [S II] 6717 / 6731 ratio increases across knot $\mathrm{P}$ (from west to east), with values of $\sim 1.25$ to 1.35 along $6^{\prime \prime}$ (see Fig. 9). The electron densities range from $200 \mathrm{~cm}^{-3}$ to $80 \mathrm{~cm}^{-3}$. The [N II] / [S II] emission line ratio first increases eastwards, and then maintains a more or less constant value of $\sim 0.40$ across the brighter regions of knots $\mathrm{P}$ and $\mathrm{Q}$.

The results found in this section therefore are:

- the radial velocities of knots I and $\mathrm{J}$ decrease from west to east, while the PV diagram of knot P shows a S-shaped structure;
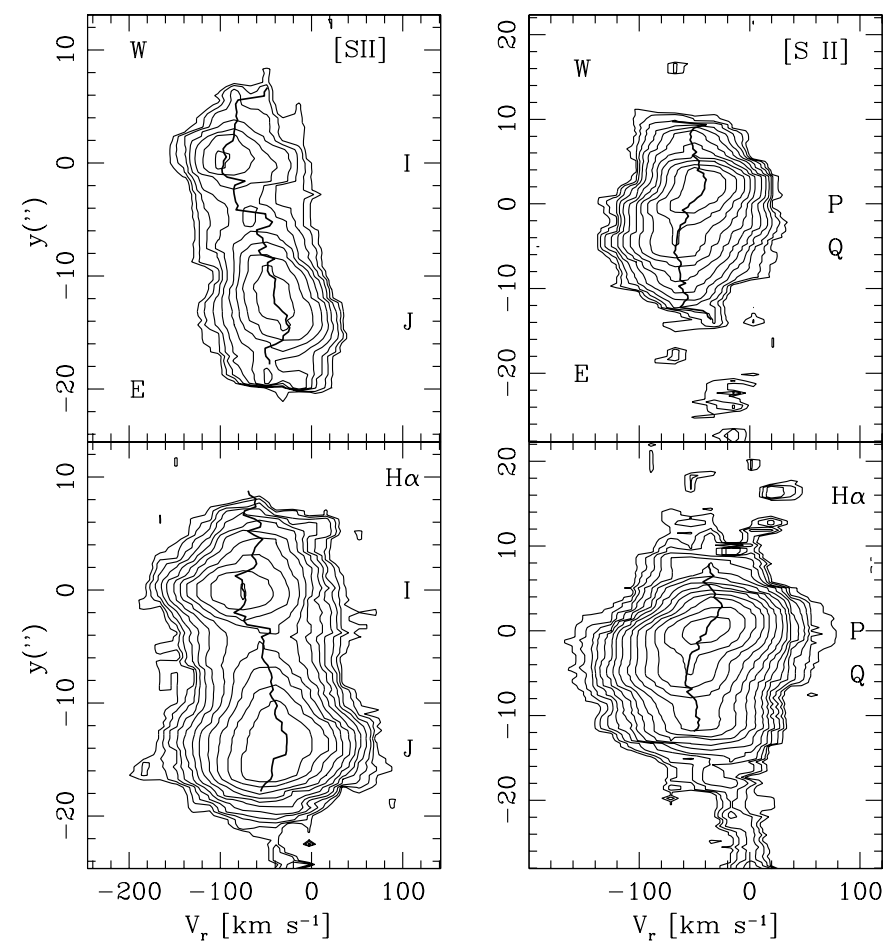

Fig. 8. $\mathrm{H} \alpha$ (bottom panels) and [S II] $6717 \AA$ (top panels) long slit spectrum across the jet at the position of knots I+J (left panels) and $\mathrm{P}+\mathrm{Q}$ (rigth panels); depicted with $2^{1 / 2}$, logarithmic contours. The superimposed solid lines are the radial velocities (with respect to the molecular cloud) corresponding to the peak of the line profiles. The angular distances are measured from the central $\mathrm{H} \alpha$ peak of knot I (left panels) and knot $\mathrm{P}$ (right panels).

- the [N II] / H $\alpha$ and [S II] / H $\alpha$ ratios increase across knots I and $\mathrm{J}$ moving from west to east, and show an overall decreasing trend across knot $\mathrm{P}$;

- the electron densities decrease from west to east across knots I, J and P;

- the $[\mathrm{N} \mathrm{II}] /[\mathrm{S} \mathrm{II}]$ emission line ratios abruptly change at the position of knot I (from a value of $\sim 0.20$ west to 0.40 east of the knot), and this ratio is more or less constant across the bright regions of knot $\mathrm{P}$.

\subsection{The spatial displacements between the $\mathrm{H} \alpha$ and [S II] spatial intensity distributions}

Noriega-Crespo et al. (1996) pointed out the differences observed between the intensity distribution of the optical emission $\left(\mathrm{H} \alpha\right.$ and $\left[\mathrm{S} \mathrm{III])} \mathrm{and} \mathrm{the} \mathrm{H}_{2}\right.$ emission along and across the axis of the HH 110 jet. An inspection of their Figs. 3, 4 and 5 also shows clear differences between the $\mathrm{H} \alpha$ and the [S II] emission lines.

In the following, we explore the differences between the spatial distributions of the $\mathrm{H} \alpha$ and [S II] velocity integrated intensities (Fig. 10) across the axis of the HH 110 jet, as obtained from the spectra with slit positions "sb"-“se" (Table 1).

In Fig. 10 (top left panel) we show the $\mathrm{H} \alpha$ and [S II] intensity distribution over the cross section of the jet at knot $\mathrm{C}$. This graph reveals the apparent spatial separation between both 


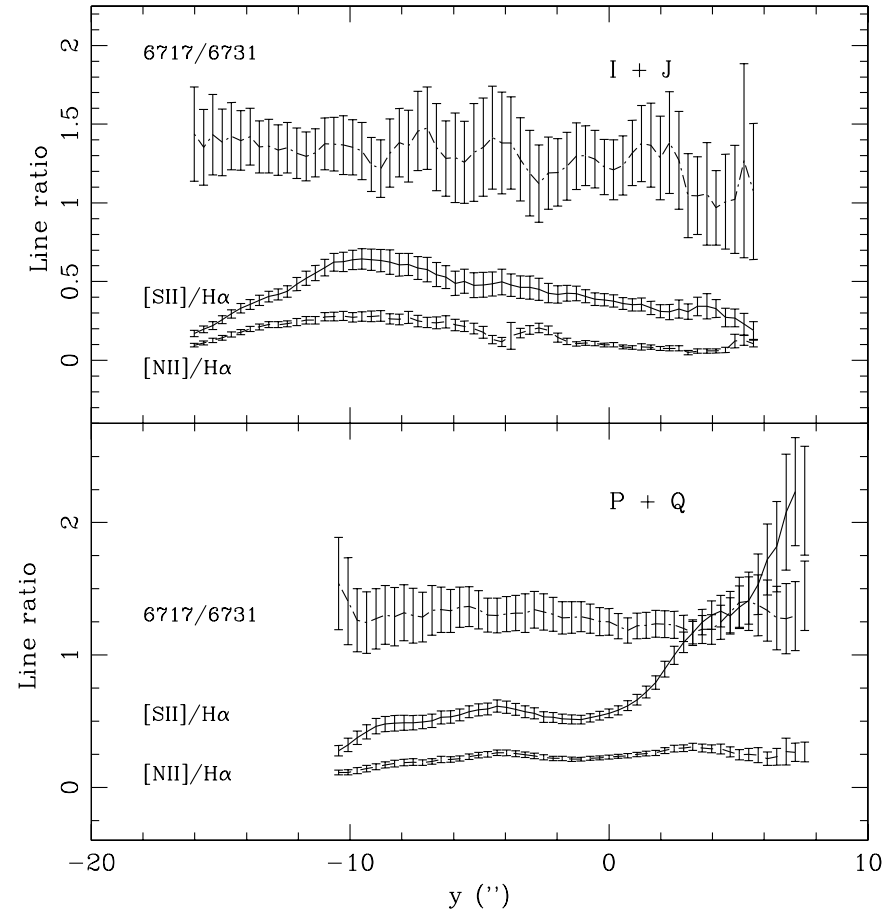

Fig. 9. [N II] $6583 / \mathrm{H} \alpha$, [S II] $(6717+6731) / \mathrm{H} \alpha$, and [S II] 6717 / 6731 line ratios through the cross section of the jet at the positions of knots $\mathrm{J}+\mathrm{I}$ (top panel), and $\mathrm{P}+\mathrm{Q}$ (bottom panel), as a function of distance from the central peak. The intensities were integrated over all the radial velocity range in which line emission was detected.

distributions. We observe a clear offset in position of the intensity peaks, with the [S II] intensity maximum at $0 .{ }^{\prime} 5$ towards the east with respect to the $\mathrm{H} \alpha$ peak. For knot B, we observe a similar spatial distribution in $\mathrm{H} \alpha$ and [S II], in agreement with the results obtained by Noriega-Crespo et al. (1996).

For knot $\mathrm{P}$, we find a displacement between the $\mathrm{H} \alpha$ and the [S II] $6717 \AA$ spatial intensity distributions (Fig. 10, top right panel). We observe a clear offset (by 0. ' 8 ) between the position of the intensity maximum seen in $\mathrm{H} \alpha$ and in [S II] $6717 \AA$. In contrast to the spatial displacement observed across knot $\mathrm{C}$, the [S II] peak is displaced towards the west with respect to the $\mathrm{H} \alpha$ peak, again in agreement with the results of Noriega-Crespo et al. (1996).

Finally, across knot $\mathbf{J}$ we observed the largest spatial offset between the $\mathrm{H} \alpha$ and [S II] spatial intensity distribution (see Fig. 10, bottom right panel), while no offset is observed across knot I. Across knot J, the [S II] peak is offset by 2.'0 westwards from the $\mathrm{H} \alpha$ peak.

The values of the observed displacements between the $\mathrm{H} \alpha$ and the [S II] $6717 \AA$ spatial intensity distributions should be taken with some caution due to the $\sim 2-3^{\prime \prime}$ angular resolution of our observations. However, we do find a good agreement between the spatial displacements shown in our spectra and the results of Noriega-Crespo et al. (1996). This agreement appears to confirm the reality of the spatial displacements which we see in our spectra.

\section{Discussion and conclusions}

As we have described in the previous sections, the $\mathrm{HH} 110$ jet has a very complex structure, with detailed, spatially dependent radial velocities, line intensities and line ratios. Let us now describe the general trends that can be deduced from the detailed structure.

The result that is most clear is that the radial velocity has a largely monotonic trend of larger (i.e., more negative) velocities as a function of position along the jet (Sect. 3.1). The velocity goes from a value of $-20 \mathrm{~km} \mathrm{~s}^{-1}$ for knot A to $-65 \mathrm{~km} \mathrm{~s}^{-1}$ in knot $\mathrm{P}$. If we consider that the jet has a mean radial velocity (with respect to the cloud) $\left\langle v_{r}\right\rangle=-42 \mathrm{~km} \mathrm{~s}^{-1}$, one can use the mean proper motion $\left\langle v_{p}\right\rangle=83 \mathrm{~km} \mathrm{~s}^{-1}$ determined by Reipurth et al. (1996), we obtained a $\phi=27^{\circ}$ between the outflow axis and the plane of the sky. On the other hand, if we consider the radial velocities and proper motions of the $\mathrm{H}, \mathrm{M}$ and $\mathrm{P}$ knots (which are the knots with the better determined proper motions) to obtain an inclination angle $\phi_{\mathrm{H}}=22^{\circ}, \phi_{\mathrm{M}}=41^{\circ}$ and $\phi_{\mathrm{P}}=38^{\circ}$, respectively. Interestingly, averaging these three values we obtain an angle of $34 \pm 8^{\circ}$ which is consistent within the errors with the $27^{\circ}$ orientation angle calculated with the mean radial velocity and proper motion (see above).

Adopting a $\phi=35^{\circ}$ angle between the outflow axis and the plane of the sky, we can deproject the measured radial velocities, and conclude that the HH 110 flow accelerates from a velocity of $35 \mathrm{~km} \mathrm{~s}^{-1}$ in knot A up to $110 \mathrm{~km} \mathrm{~s}^{-1}$ in knot P (knot A and $\mathrm{P}$ being separated by $130^{\prime \prime}$ ). This acceleration could be the result of a time-dependent ejection velocity from the outflow source (with ejection velocity decreasing as a function of time). Another possible explanation for such a velocity vs. position trend has been suggested by Raga et al. (2002), who modeled HH 110 as a wake left behind by a short-lived deflected jet, which is pinched off as the incident jet starts to penetrate the dense cloud which produced the jet deflection.

The radial velocity cross sections of the jet (see Sects. 3.2 and 3.3), show an almost constant velocity for knots B and $\mathrm{C}$, a velocity change from $\mathrm{W}$ to $\mathrm{E}$ of $\sim 60 \mathrm{~km} \mathrm{~s}^{-1}$ (corresponding to a deprojected value of $\sim 100 \mathrm{~km} \mathrm{~s}^{-1}$ ) for knots I and $\mathrm{J}$, and a more complicated, S-shaped cross section spanning a $\sim 30 \mathrm{~km} \mathrm{~s}^{-1}$ velocity range (corresponding to a deprojected value of $\sim 50 \mathrm{~km} \mathrm{~s}^{-1}$ ) for knot $\mathrm{P}$.

The [S II] line ratio along the HH 110 jet shows a more or less monotonic drop of the electron density in the downstream direction (see Sect. 3.1). The [S II] / $\mathrm{H} \alpha$ ratio first grows, reaching a maximum of $\sim 0.8$ in knots $\mathrm{H}-\mathrm{M}$, and then drops along the jet axis. The $[\mathrm{N} \mathrm{II}] / \mathrm{H} \alpha$ ratio has a qualitatively similar behaviour, showing a maximum in knot $\mathrm{M}$. The [N II] / [S II] ratio shows a more or less monotonic growth down the jet axis.

The [S II] line ratio across the jet (see Sect. 3.3) shows that across knots B and C we have a dense $\left(\sim 450-700 \mathrm{~cm}^{-3}\right)$, central region of the jet beam, with lower density $\left(\sim 100-300 \mathrm{~cm}^{-3}\right)$ regions on both sides. In knots I, J and $\mathrm{P}$, the electron density grows monotonically from $\mathrm{E}$ to $\mathrm{W}$ (from $\sim 50$ to $200 \mathrm{~cm}^{-3}$ ). The other measured line ratios show quite complex cross sections.

If we compare the cross sections of the emission of the different lines, we find that the maxima sometimes coincide (knot B and I), but that in other cases there are shifts 


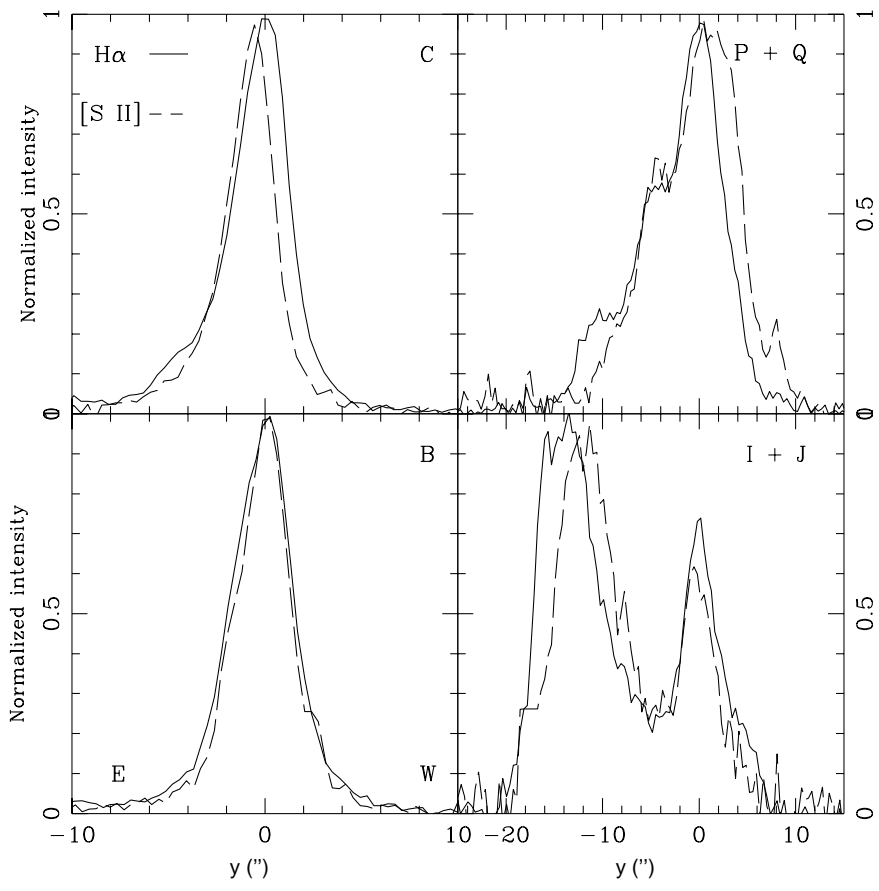

Fig. 10. Spatial $\mathrm{H} \alpha$ (solid line) and [S II] (dashed line) intensity distributions through the cross section of the jet at the location of knots B, $\mathrm{C}, \mathrm{I}+\mathrm{J}$ and $\mathrm{P}+\mathrm{Q}$. The zero point of the spatial axis is defined in each knot at the position of the peak intensity of $\mathrm{H} \alpha$. The distributions have been normalized by their peak intensities.

of $\sim 0.5-2^{\prime \prime}$ between the maxima of $\mathrm{H} \alpha$ and [S II] (knots $\mathrm{C}$, $\mathrm{J}$ and P, see Sect. 3.4).

From the above discussion, we see that while there are systematic trends along the $\mathrm{HH} 110$ jet for both the radial velocities and the emission line ratios, no clear trends are seen across the width of the jet beam. The cross sections of the jet (taken at different positions along the HH 110 jet) show complex radial velocity and line emission structures, which differ quite strongly from each other.

Given the complex structures that are observed, it does not appear to be reasonable to compare them with simple models of plane-parallel shock waves, or with "mixing-length" models of turbulent mixing layers. For example, the analytic turbulent mixing layer model of Noriega-Crespo et al. (1996) predicts that there should be a systematic velocity increase from $\mathrm{W}$ to $\mathrm{E}$ across the section of the HH 110 flow. Also, the model predicts that there should be a systematic $\mathrm{W}$ to $\mathrm{E}$ increase in the excitation of the emitted spectrum. Such systematic trends might be partially present in our data, but they mostly show complex cross sections which differ from each other at different positions along the HH 110 flow. For example, we do see that the [N II] / [S II] ratio increases from W to E, and we detect spatial offsets between the maxima of the $\mathrm{H} \alpha$ and [S II] emission cross sections, in qualitative agreement with the mixing layer model of Noriega-Crespo et al. (1996). However, we do not see clear trends in the radial velocity across the HH 110 flow.
We should note that one might expect to have large discrepancies between observations of a turbulent flow (which would correspond to a "snapshot" of the turbulent eddies) and the predictions from a "mixing length" turbulent mixing layer model (which attempts to describe the properties of a smooth, ensemble-averaged "mean flow"). Therefore, it is difficult to use comparisons between observations and this type of models to prove or disprove whether the emission from $\mathrm{HH} 110$ is indeed formed in a turbulent mixing layer.

A possible approach would be to compute $3 \mathrm{D}$ models of a jet/cloud interaction similar to the ones of Raga et al. (2002), but including a description of the S II and N II ions, so as to be able to obtain predictions of the observed emission lines. From such a simulation, one could explore whether or not the complex properties which we have discussed above are consistent with a jet/cloud collision scenario.

As noted by Raga et al. (2002), a model of a jet/cloud collision does produce a "pinched-off" deflected jet beam that has properties which resemble the $\mathrm{H} \alpha$ and $\mathrm{H}_{2}$ 1-0 s(1) emission of HH 110. Such a model also produces an apparent "acceleration" along the flow, which is the characteristic signature of the wake left behind the pinched-off deflected jet beam. Our data do show this acceleration, which is also seen in the proper motions of Reipurth et al. (1996).

The spectroscopic data presented in this paper, however, give a much more detailed description of the kinematics and excitation of HH 110. It will be necessary to compute new models (with higher resolution and a more detailed treatment of the atomic/ionic processes) in order to see whether or not these observations agree in detail with the predictions from a jet/cloud interaction model.

Acknowledgements. The work of RE, RL and AR was supported by CGICYT grant PB 98-0670-C02 and MCyT grant AYA2002-00205 (Spain). The work of GA was supported by CGICYT grant PB 980670-C02 (Spain). RE and AR acknowledges support from the ACI 2000-8 Direcció General de Recerca (Spain). GA acknowledges support from Junta de Andalucía (Spain). The work of ACR was supported by CONACyT grants 34566-E and 36572-E.

\section{References}

Carter, D., Benn, C. R., Rutten, R. G. M., et al. 1994, WHT ISIS user's Manual, Isaac Newton Group Telescopes, Royal Greenwich Observatory

Choi, M. 2001, ApJ, 550, 817

Davis, C. J., Mundt, R., \& Eislöffel, J. 1994, ApJ, 437, L55

Noriega-Crespo, A., Garnavich, P. M., Raga, A. C., Cantó, J., \& Böhm, K.-H. 1996, ApJ, 462, 804

Raga, A. C., \& Cantó, J. 1995, Rev. Mex. Astron. Astrofis., 31, 51

Raga, A. C., de Gouveia Dal Pino, E. M., Noriega-Crespo, A., Mininni, P. D., \& Velázquez, P. F. 2002, A\&A, 392, 267

Reipurth, B., \& Olberg, M. 1991, A\&A, 246, 535

Reipurth, B., Raga, A. C., \& Heathcote, S. 1996, A\&A, 311, 989

Rodríguez, L. F., Reipurth, B., Raga, A. C., \& Cantó, J. 1998, Rev. Mex. Astron. Astrofis., 34, 69

Sepúlveda, I. 2001, Ph.D. Thesis, Univ. Barcelona 